\title{
Spectrum Allocation by Sealed Bid Game Theory
}

\author{
Aritra De, Tirthankar Datta
}

\begin{abstract}
Wireless communication subscribers are increasing day by day specially in fifth generation (5G) wireless communication where multiple number of users (Multiple Input Multiple Output or MIMO) can be served in a specific time. The heavy data usage is also enhanced with the increasing the number of subscribers, this data transfer speed depends on the amount of spectrum allocation to the specific subscriber. Thus, spectrum allocation is a major criterion for wireless communication performance improvement. The spectrum allocation efficiency can be observed by Game Theory, which is a popular decision maker of modern era. Sealed Bid Game theory is one of the popular segment of the game theory. The spectrum allocation can be done by using Sealed Bid Game theory and spectrum equilibrium can be observed by using different sub division of Sealed Bid Game theory.
\end{abstract}

Keywords:5G, MIMO, Game Theory, Sealed Bid Game Theory, Spectrum Allocation.

\section{INTRODUCTION}

Wireless communication spectrum allocation is major criteria for the performance improvement of the system [1]. Multiple number of subscribers can be served by modern mobile communication generation [2].The multiple number of subscribers data speed and voce quality can be improved by using efficient spectrum allocation technique [3].The spectrum can be done by simulation method or experimental method [4].

The spectrum also can be done by using different optimization technique [5].The optimization technique is difficult to understand and mathematical calculation is time consuming [6].

Game Theory is less time consuming and it is used heavily in the modern era[7].Game theory does not assume any knowledge of its players[8].The only way to appreciate game theory is to see it in action, or better still to put in into action[9]. [10].

The user of the mobile subscriber can be static or dynamic

Revised Manuscript Received on December 30, 2019.

* Correspondence Author

Aritra De.* is with MAKAUT INDIA. He is now with the Department of Electronics \& Communication Engineering, as a Research Scholar

Tirthankar Datta is with the Department of Electronics \& Communication Engineering, Netaji Subhash Engineering College INDIA, as a Professor and Principal

(C) The Authors. Published by Blue Eyes Intelligence Engineering and Sciences Publication (BEIESP). This is an open access article under the CC BY-NC-ND license (http://creativecommons.org/licenses/by-nc-nd/4.0/)
In this work is done by considering spectrum allocation to two static user as shown in the figure (1) or with a macro cell in fifth generation wireless communication as shown in the figure (2) or spectrum allocation to a moving user[11].

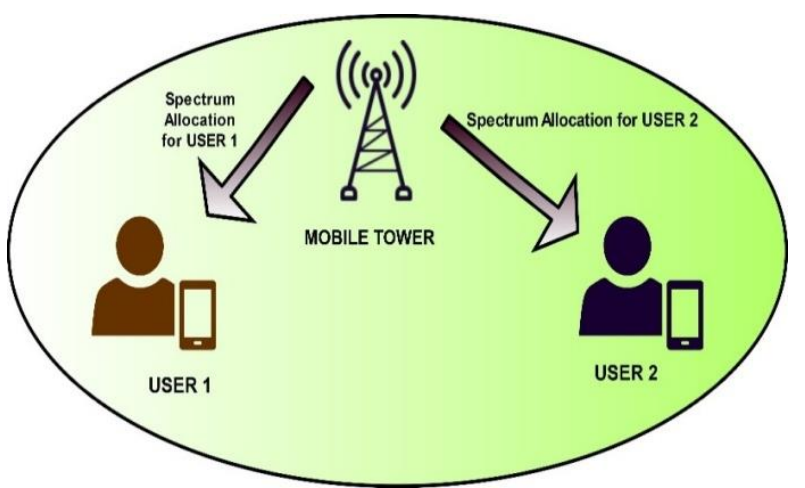

Figure 1: Spectrum Allocation for the Mobile User

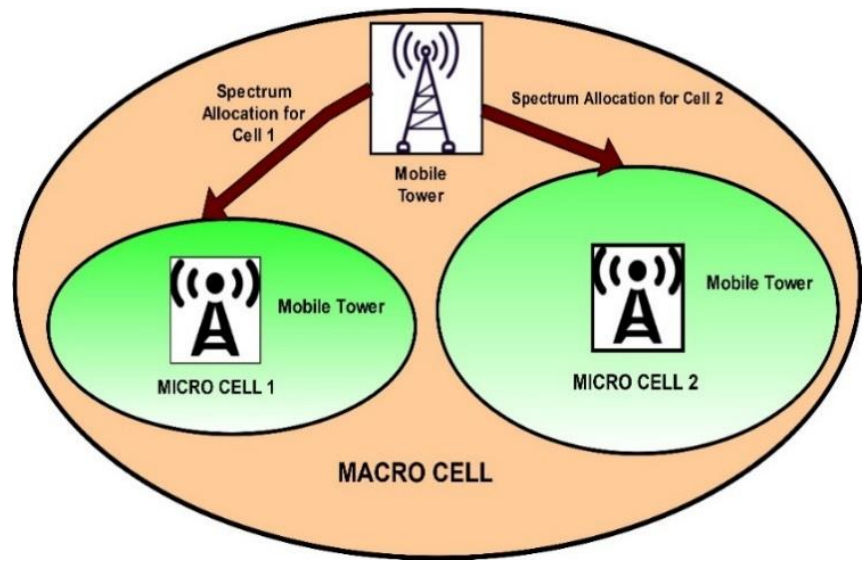

Figure 2: Spectrum Allocation for the Micro Cell

In this work wireless generation is compared in the section II, Bayesian second price auction game theory with average value calculation is discussed in the section III and IV respectively, sealed bid first price auction is discussed with average value calculation is discussed in the section V and VI respectively, two player all pay auction is discuss with average value calculation is discussed in the section VII and VIII respectively. Conclusion of the work is discussed in the section IX. 


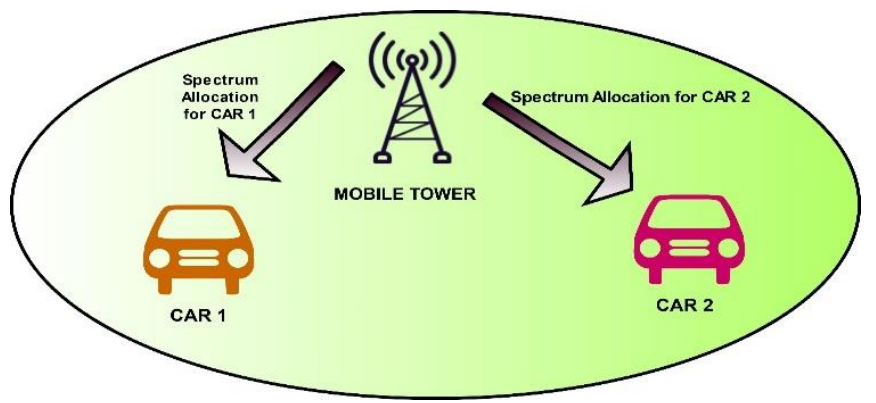

Figure 3: Spectrum Allocation for the Moving User

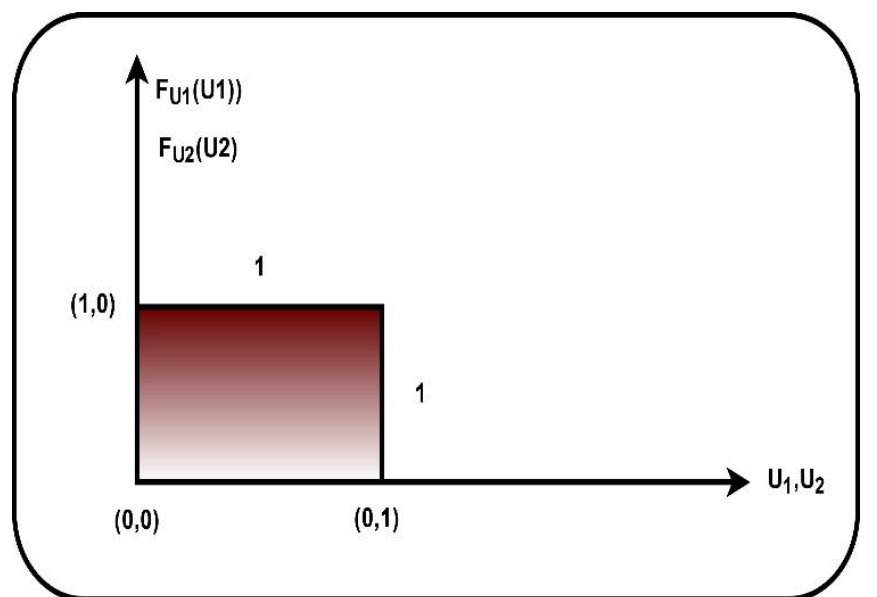

Figure 4: Probability Density Function of Spectrum allowed (sealed bid first price auction)

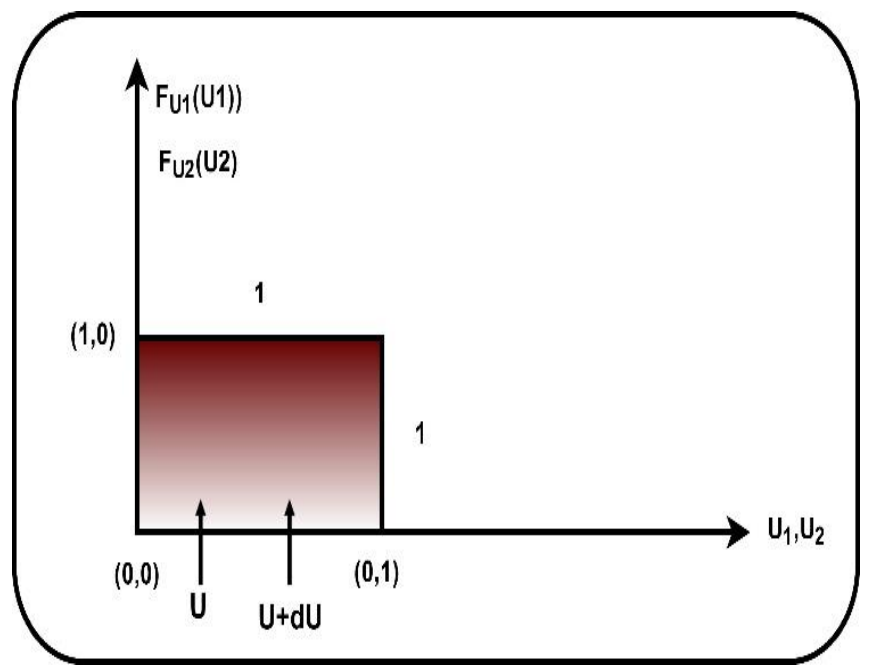

Figure 5: Probability Density Function of Spectrum allowed (extended revenue of the first price auction)

\section{WIRELESS GENERATION COMPARISON}

Table 1: Mobile generation comparison

\begin{tabular}{|c|c|c|c|c|c|}
\hline Feature & $1 \mathrm{G}$ & $2 G$ & $3 G$ & $4 G$ & $5 G$ \\
\hline $\begin{array}{l}\text { Deploymen } \\
t\end{array}$ & 1980 & 1990 & 2001 & 2010 & $\begin{array}{l}2020 \\
\text { and } \\
\text { beyond }\end{array}$ \\
\hline $\begin{array}{l}\text { Frequency } \\
\text { Band }\end{array}$ & $\begin{array}{l}800 \\
\mathrm{MHz}\end{array}$ & $\begin{array}{l}900 \\
\mathrm{MHz}\end{array}$ & $\begin{array}{l}2100 \\
\mathrm{MHz}\end{array}$ & $\begin{array}{l}2600 \\
\mathrm{MHz}\end{array}$ & $\begin{array}{l}\text { 3-90GH } \\
\mathrm{z}\end{array}$ \\
\hline Speed & $\begin{array}{l}2 \\
\text { kbps }\end{array}$ & $\begin{array}{l}64 \\
\text { kbps }\end{array}$ & $\begin{array}{l}\text { 2Mbp } \\
\mathrm{s}\end{array}$ & $\begin{array}{l}1 \mathrm{~Gb} \\
\mathrm{ps}\end{array}$ & $\begin{array}{l}\text { Higher } \\
\text { than } \\
\text { 1Gbps }\end{array}$ \\
\hline
\end{tabular}

\section{BAYESIAN SECOND PRICE AUCTION}

The spectrum is allocated to two user $Q_{1}$ and $Q_{2}$.The spectrum is allocated to the user $Q_{1}$ is $C_{1}$ and maximum spectrum is allowed is $U_{1}$, the spectrum is allocated to the user $Q_{2}$ is $C_{2}$ and maximum spectrum is allowed is $U_{2}$.

If $C_{1} \geq C_{2}$ then $Q_{1}$ will win else if $C_{2}>C_{1}$ then $Q_{2}$ wins so it mean player with highest bid wins the auction.

If $C_{1} \geq C_{2}$ then $Q_{1}$ will win and pays the second highest bid $C_{2}$.

if $C_{2}>C_{1}$ then $Q_{2}$ wins and pays the second highest bid $C_{1}$.

Now each and every player has a private valuation, consider the valuation of $Q_{1}, Q_{2}$ is $U_{1}, U_{2}$ respectively.

$U_{1}, U_{2}$ are independent, distributed and random variable as shown in figure (4).

The Nash Equilibrium of second price auction is

$C_{1}=U_{1}$.

$C_{2}=U_{2}$

Now start with the assumption that

$\mathrm{C}_{2}=\mathrm{U}_{2}$

\section{A. Case 1}

Consider $U_{1} \geq U_{2}$, the bidding of player 2 is $C_{2}=U_{2}$ also if $\mathrm{C} \geq U_{2}, Q_{1}$ wins the auction and pays the second highest bid $C_{2}=U_{2}$. Net payoff $=U_{1}-U_{2} \geq 0$.

If he bids, $\mathrm{C} \leq C_{2}=U_{2}$ then player $Q_{1}$ loses the auction and his net payoff is 0 .

Therefore, any bid $\mathrm{C} \geq U_{2}$ is a best response that mean $\mathrm{C}=U_{1}$ is a best response.

\section{B. Case 2}

If $U_{1} \leq U_{2}$ player $Q_{2}$ is bidding $C_{2}=U_{2}$.If $Q_{1}$ bids $\mathrm{C} \geq$ $C_{2}=U_{2}$,then he wins the auction and pays second highest bid $C_{2}=U_{2}$. Net payoff $=U_{1}-U_{2} \leq 0$.

If he bids $\mathrm{C}<C_{2}=U_{2}$ then he loses the auction and his payoff is 0 .Therefore any bid $\mathrm{C}<C_{2}=U_{2}$ is a best response. In particular $\mathrm{C}=U_{1}$ is a best response.

If player $Q_{2}$ is bidding $C_{2}=U_{2}$, then $C_{1}=U_{1}$ is a best response for player $Q_{1}$.

Similarly it can be shown that if $Q_{1}$ is bidding $C_{1}=U_{1}$ then $\mathrm{C}=U_{2}$ is a best response for $Q_{2}$.

Hence the Nash equilibrium of second price auction is

$C_{1}=U_{1}$

$C_{2}=U_{2}$

So, each player bidding his true valuation is the Nash equilibrium for the second price auction.

\section{EXPECTED REVENUE OF SECOND PRICE AUCTION}

$C_{1}=U_{1}$

$C_{2}=U_{2}$

Noted that $U_{1} \geq U_{2}$ then $C_{1} \leq C_{2}$,so $Q_{1}$ wins the auction and pays second highest bid $C_{2}=U_{2}$, if $U_{1} \geq U_{2}$, revenue $=U_{2}$.

If $U_{1}<U_{2}$ then $C_{1}>C_{2}$, so $Q_{2}$ wins the auction and pays second highest bid $U_{1}$, therefore revenue $=U_{1}$.

So, it can be concluded that the revenue to the auctioneer in the Bayesian second price auction is minimum $\left\{U_{1}, U_{2}\right\}$.

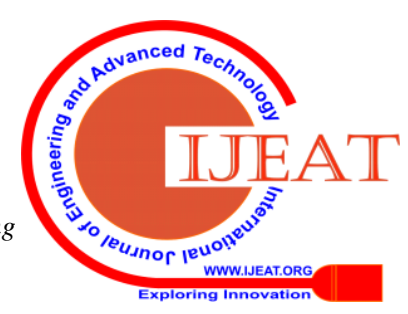


$U_{1}, U_{2}$ are independent and Probability Density Function (PDF) $F_{U_{1}}\left(U_{1}\right)$ and $F_{U_{2}}\left(U_{2}\right)$ of Maximum spectrum allowed is distributed uniformly in the interval $[0,1]$ as shown in the figure 5 .

A. Case 1

$U_{1} \leq U_{2}$

$U_{1}$ is lies in [U,U $\left.+\mathrm{dU}\right]$

$U_{2}$ is lies in [U+dU,1]

$P_{r}=P_{r}\left(U_{1} \in[U, U+d U]\right) \times P_{r}\left(U_{2} \in[U+d U, 1]\right)$

$=\mathrm{dU} \times(1-\mathrm{U}-\mathrm{dU})$

$=\mathrm{dU}(1-\mathrm{U})$

$=(1-\mathrm{U}) \mathrm{dU}$

B. Case 2

$U_{1}>U_{2}$

$U_{1}$ is lies in [U+dU,1]

$U_{2}$ is lies in [U,U+dU]

$P_{r}=P_{r}\left(U_{2} \in[U, U+d U]\right) \times P_{r}\left(U_{1} \in[U+d U, 1]\right)$

$=\mathrm{dU} \times(1-\mathrm{U}-\mathrm{dU})$

$=\mathrm{dU}(1-\mathrm{U})$

$=(1-\mathrm{U}) \mathrm{dU}$

The total probability minimum $\left\{U_{1}, U_{2}\right\}$ lies in between the interval [U,U+dU\} is $2(1-\mathrm{U})$.

Revenue to the auctioneer=minimum $\left\{U_{1}, U_{2}\right\}$

Since minimum lies in $[\mathrm{U}, \mathrm{U}+\mathrm{dU}]$,revenue $=\mathrm{U}$. Expected revenue $=P_{r} \times \mathrm{U}$

$=2(1-\mathrm{U}) \mathrm{dU}$.....

Expected revenue

$=\int_{0}^{1} 2(1-U) u d U$

$=1 / 3$.

Hence expected revenue $=1 / 3$ also the revenue is independent.

\section{SEALED BID FIRST PRICE AUCTION}

The spectrum is allocated to two user $Q_{1}$ and $Q_{2}$.The spectrum is allocated to the user $Q_{1}$ is $C_{1}$ and maximum spectrum is allowed is $U_{1}$, the spectrum is allocated to the user $Q_{2}$ is $C_{2}$ and maximum spectrum is allowed is $U_{2}$.

The Probability Density Function (PDF) $F_{U_{1}}\left(U_{1}\right)$ and $F_{U_{2}}\left(U_{2}\right)$ of Maximum spectrum allowed is distributed uniformly in the interval $[0,1]$ as shown in the figure 4.

The Game Theory bidding strategy is as following

$C_{1}=\frac{1}{2} U_{1}$

$C_{2}=\frac{1}{2} U_{2}$

$\pi(\mathrm{C})$ Denotes the payoff to the user $Q_{1}$ as a function of $\mathrm{C}$ where $C$ is maximum spectrum allocated to the mobile tower.

User $Q_{1}$ wins the auction game i.e. $\mathrm{C} \geq C_{2}$, then the payoff is $=$ Valuation of the user 1-Bid paid on winning the auction

$=U_{1}-\mathrm{C}$.

Average payoff to player 1 is given by,

$P_{r}($ win $) \times\left(U_{1}-\mathrm{C}\right)+P_{r}($ loss $) \times 0$

So,

$\pi(\mathrm{C})=P_{r}($ win $) \times\left(U_{1}-\mathrm{C}\right)$

The winning condition for the player 1 is

$\mathrm{C} \geq C_{2}=\frac{1}{2} U_{2}$

$\mathrm{C} \geq \frac{1}{2} U_{2}$

$U_{2} \leq 2 \mathrm{C}$
Since $U_{2}$ is distributed uniformly in $[0,1], U_{2}$ must have in [0,2C]

Probability $U_{2}$ lies in $[0,2 \mathrm{C}]$

$=\int_{0}^{2 C} F_{U_{2}}\left(U_{2}\right) \mathrm{d} U_{2}$

$=\int_{0}^{2 C} \mathrm{~d} U_{2}$

$=\left.U_{2}\right|_{0} ^{2 C}$.

$=2 \mathrm{C}$.

Hence, $P_{r}($ win) for player 1 is $2 \mathrm{C}$,therefore

$\pi(\mathrm{C})=P_{r}($ win $) \times\left(U_{1}-\mathrm{C}\right)$.

$=2 \mathrm{C} \times\left(U_{1}-\mathrm{C}\right)$

$\pi(\mathrm{C})=2 \mathrm{C} U_{1}-2 C^{2}$

$\frac{d \pi(C)}{d C}=2 U_{1}-4 \mathrm{C}=0$.

$C^{*}=\frac{1}{2} U_{1}$

So, If $C_{2}=\frac{1}{2} U_{2}$, then the bid $C_{1}=\frac{1}{2} U_{1}$ is the best response for the user 1.

By following the same procedure it can be proved that if $C_{1}=\frac{1}{2} U_{1}$ then $C_{2}=\frac{1}{2} U_{2}$ is the best response for the user 2 .

\section{EXPECTED REVENUE OF THE FIRST PRICE AUCTION}

Nash equilibrium is given by

$C_{1}=\frac{1}{2} U_{1} \ldots$
$C_{2}=\frac{1}{2} U_{2}$

Now the player wins who called for maximum bid. Hence

Revenue=maximum $\left\{C_{1}, C_{2}\right\}$. .

$=$ maximum $\left\{\frac{1}{2} U_{1}, \frac{1}{2} U_{2}\right\}$

$=\frac{1}{2}$ maximum $\left\{U_{1}, U_{2}\right\}$

$U_{1}, U_{2}$ are uniform distributed in $[0,1]$ and probability for equation (21) lies in the infinitesimal interval [U,U+dU] as shown in the figure (4).

\section{A. Case 1}

$U_{1}$ is the maximum $U_{1}$ lies in [ $\left.\mathrm{U}, \mathrm{U}+\mathrm{dU}\right]$ and $U_{2}$ lies in $[0, \mathrm{U}]$

So,

$P_{r}=P_{r}\left(U_{1} \in[U, U+d U]\right) \times P_{r}\left(U_{2} \in[0, U]\right)$

$=\mathrm{dU} \times \mathrm{U}$.

$=\mathrm{UdU}$

\section{B. Case 2}

$U_{2}$ is the maximum $U_{2}$ lies in [ $\left.\mathrm{U}, \mathrm{U}+\mathrm{dU}\right]$ and $U_{1}$ lies in $[0, \mathrm{U}]$

So,

$P_{r}=P_{r}\left(U_{1} \in[0, U]\right) \times P_{r}\left(U_{2} \in[U, U+d U]\right)$

$=\mathrm{U} \times \mathrm{dU}$

$=\mathrm{UdU}$

\section{Equations}

Probability that maximum $\left\{U_{1}, U_{2}\right\}$ is lies in [U,U+dU] $=\mathrm{UdU}+\mathrm{UdU}$.

$=2 \mathrm{UdU}$.

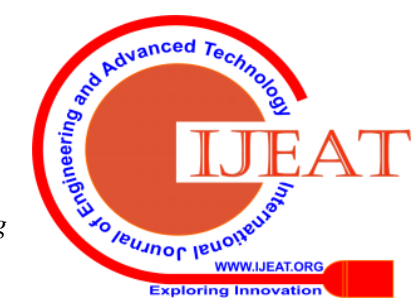


So, Average revenue corresponding to maximum $\left\{U_{1}, U_{2}\right\} \in[\mathrm{U}, \mathrm{U}+\mathrm{dU}]$

$=\frac{1}{2} \mathrm{U} \times 2 \mathrm{UdU}$

$=U^{2} \mathrm{dU}$

So, total average revenue to the auctioneer is

$=\int_{0}^{1} U^{2} \mathrm{dU}$.

$=\left.\frac{1}{3} U^{3}\right|_{0} ^{1}$

$=\frac{1}{3}$.

The expected revenue of the auctioneer is $=1 / 3$.

\section{TWO PLAYER ALL PAY PRICE AUCTION}

The spectrum is allocated to two user $Q_{1}$ and $Q_{2}$.The spectrum is allocated to the user $Q_{1}$ is $C_{1}$ and maximum spectrum is allowed is $U_{1}$, the spectrum is allocated to the user $Q_{2}$ is $C_{2}$ and maximum spectrum is allowed is $U_{2}$.

User with highest spectrum allocation wins the game. Both the player pay their bid irrespective of their outcome.

Now, assume that $U_{1}$ and $U_{2}$ denotes the valuations of $Q_{1}$ and $Q_{2}$.

The Probability Density Function (PDF) $F_{U_{1}}\left(U_{1}\right)$ and $F_{U_{2}}\left(U_{2}\right)$ of Maximum spectrum allowed is distributed uniformly in the interval $[0,1]$ as shown in the figure 4 .

The Nash Equilibrium is as following

$C_{2}=1 / 2 U_{2}^{2}$

Now, assume that player $Q_{2}$ is bidding $C_{2}=1 / 2 U_{2}^{2}$, also assume player $Q_{1}$ bids C.

$\pi(\mathrm{C})$ is expected payoff to player 1 as a function $\mathrm{C}$.

$\pi(\mathrm{C})=P_{r}($ win $) \times\left(U_{1}-\mathrm{C}\right)+P_{r}($ loss $) \times(-\mathrm{C})$.

$Q_{1}$ will win if $\mathrm{C} \geq C_{2}=1 / 2 U_{2}^{2}$

$=1 / 2 U_{2}^{2} \leq \mathrm{C}$.

$U_{2} \leq \sqrt{2 C}$

$P_{r}($ win $)=P_{r}\left(U_{2} \leq \sqrt{2 C}\right)$

$P_{r}$ (win) $=P_{r}\left(U_{2} \in[0, \sqrt{2 C})\right.$.

$P_{r}$ (loss) $=1-P_{r}$ (win)

$P_{r}($ loss $)=1-\sqrt{2 C}$.

So, from equation (34) it can be derived that

$\pi(\mathrm{C})=\sqrt{2 C}\left(U_{1}-\mathrm{C}\right)+(1-\sqrt{2 C}) *(-\mathrm{C})$.

$\pi(\mathrm{C})=\sqrt{2 C} U_{1}-\mathrm{C}$.

By differentiating equation (43) with respect to $\mathrm{C}$

$\frac{\partial \pi(\mathrm{C})}{\partial C}=\sqrt{2 C} U_{1} * \frac{1}{\sqrt{2 C}}-1=0$.

$\mathrm{C}=1 / 2 U_{1}^{2}$.

Similarly, it can be shown that if $C_{1}=1 / 2 U_{1}^{2}$, then $\mathrm{C}=1 / 2 U_{2}^{2}$ is a best response bid for user $Q_{2}$.

$C_{1}=1 / 2 U_{1}^{2}$
$C_{2}=1 / 2 U_{2}^{2}$

So, equation (46) and (47) are Nash Equilibrium.

VIII. EXPECTED REVENUE OF TWO PLAYER ALL PAY PRICE AUCTION

Revenue $=C_{1}+C_{2}$.

$=\frac{1}{2} U_{1}^{2}+\frac{1}{2} U_{2}^{2}$

Expected Revenue

$=\frac{1}{2} E\left\{U_{1}^{2}\right\}+\frac{1}{2} E\left\{U_{2}^{2}\right\}$. . .

$=\frac{1}{2} \int_{0}^{1} U_{1}^{2} \mathrm{~d} U_{1}+\frac{1}{2} \int_{0}^{1} U_{2}^{2} \mathrm{~d} U_{2}$.
$C_{1}=1 / 2 U_{1}^{2}$

$=\frac{1}{2} \int_{0}^{1} U_{1}^{2} F_{U_{1}}\left(U_{1}\right) \mathrm{d} U_{1}+\frac{1}{2} \int_{0}^{1} U_{2}^{2} F_{U_{2}}\left(U_{2}\right) \mathrm{d} U_{2}$

$=\left.\frac{1}{2} \frac{U_{1}^{3}}{3}\right|_{0} ^{1}+\left.\frac{1}{2} \frac{U_{2}^{3}}{3}\right|_{0} ^{1}$.
$=\frac{1}{2} * \frac{1}{3}+\frac{1}{2} * \frac{1}{3} \ldots \ldots \ldots$
$=\frac{1}{3} \ldots \ldots \ldots \ldots \ldots \ldots$

Equation (55) is the revenue

\section{CONCLUSION}

This work is completed by using different bidding game theoretical approach. The Nash equilibrium is calculated in each sub part of sealed bid game theory, which is nothing but best approach of spectrum allocation.

\section{ACKNOWLEDGMENT}

This work is acknowledged to National Program Technical Enhanced Learning (NPTEL) an initiative by Government of INDIA (GOI).This work is dedicated to Prof (Dr.)Vimal Kumar and Prof(Dr.)Aditya K.Jagannatham of Indian Institute of Technology, Kanpur for their impressive lecture series in NPTEL.

\section{REFERENCES}

1. Zhi Chen ; Xinying Ma ; Bo Zhang ; Yaxin Zhang ; ZhongqianNiu ; NingyuanKuang ; Wenjie Chen ; Lingxiang Li ; Shaoqian Li, "A survey on terahertz communications, "China Communications 08 March 2019, pp. 1-35.

2. AritraDe ;TirthankarDatta“"Improvement of Performance of MIMO System Using Different Protocols” Fifteenth International Conference on Wireless and Optical Communications Networks (WOCN), pp. 1-5.

3. AritraDe ;TirthankarDatta "Some Aspects of Massive MIMO Spectrum Sharing" Fifteenth International Conference on Wireless and Optical Communications Networks (WOCN), pp.34-39.

4. Lifeng. Wang, Hien. Quoc Ngo, Magnad. Elkashlan, Trung Q. Duong, Kai-Kit. Wong, "Massive MIMO in spectrum haring networks: Achievable rate and power efficiency", IEEE System Journal, vol. 11, no. 1, pp. 20-31, March. 2017.

5. Y Y Li, N Li, H Li, W Xie et al., "Spectrum Sharing Based on Overlay Cognitive Full-Duplex Two-Way OFDM Relaying", IEEE. T. Veh. Technol, vol. 67, pp. 2324-2334, 2017.

6. H Li, X Zhao, "Joint resource allocation for OFDM-based cognitive two-way multiple AF relays networks with imperfect spectrum sensing", IEEE. T. Veh. Technol, vol. 67, pp. 6286-6300, 2018.

7. Bond et al., "A game theory perspective on environmental assessment: What games are played and what does this tell us about decision making rationality and legitimacy?", Environmental Impact Assessment Review, vol. 57, pp. 187-194, Feb. 2016.

8. F.F. Folami, "Gender Inequality and Role-strained among Male Nursing Students in Selected Nursing Institution Lagos Nigeria", Journal of Education and Training Studies, vol. 5, pp. 214-219, Jun. 2017.

9. William Poundstone, Prisoner's Dilemma/John von Neumann Game Theory and the Puzzle of the Bomb, Anchor, 1993.

10. A. Ali, W. Hamouda, "Advances on spectrum sensing for cognitive radio networks: theory and applications", IEEE Commun. Surv. Tutorials, vol. 19, no. 2, pp. 1277-1304, 2017.

11. A. Nath, N. Sarma, "A distributed solution for cooperative spectrum sensing scheduling in multiband cognitive radio networks", J. Netw. Comput. Appl., vol. 94, pp. 69-77, 2017.

\section{AUTHORS PROFILE}

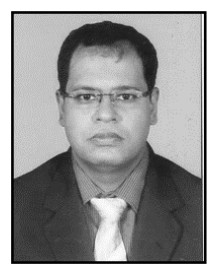

Aritra De received his B. Tech from Techno India, Salt Lake, Kolkata MTech from Institute of Engineering \& Management, Salt Lake Kolkata. He has 9years of teaching experience. He is now pursuing his $\mathrm{PhD}$ from the MaulanaAbulKalam Azad University of Technology formerly known as the West Bengal University Technology.

Published By: 


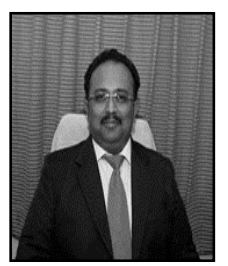

TirthankarDattareceived his $\mathrm{PhD}$ (Engg.) Degree in Microelectronics \& Nano Technology under the Department of Electronics \& Telecommunication Engineering of Jadavpur University. Prior to that, he received M. Tech. Degree in Radio Physics \& Electronics from the Institute of Radio Physics and Electronics,University of Calcutta.He is associated with Netaji Subhash Engineering College(NSEC)as Engineering Department and Principal. Professor of Electronics \& Communication 